\title{
Presbyphagia to Dysphagia: Multiple Perspectives and Strategies for Quality Care of Older Adults
}

\author{
Ashwini M. Namasivayam-MacDonald, Ph.D., CCC-SLP, SLP(C) ${ }^{1}$ and \\ Luis F. Riquelme, Ph.D., CCC-SLP, BCS-S ${ }^{2,3}$
}

The aging population is rapidly growing, requiring speechlanguage pathologists to better manage a caseload that includes older adults who have a variety of needs. The purpose of this review is to summarize and discuss the current available evidence that will allow speech-language pathologists to make informed clinical decisions when working with older adults. To facilitate this, this article first establishes an understanding of both normal and disordered swallowing physiology in older adults, including how to differentiate between functional changes to swallowing (presbyphagia) and dysphagia. Other important factors to consider, such as caregiver burden, are also discussed so that clinicians can learn how to best support aging in place. Best practices for screening both community-dwelling older adults and residents of longterm care are identified as part of a framework introduced to guide decision making. The critical components of clinical swallow assessments are reviewed, including the adoption of an ethnographic approach and why nutritional status, urinary tract infections, and delirium are important considerations when working with older adults. Factors contributing to, and associated with, aspiration and aspiration pneumonia are also discussed so that clinicians better understand how to take a comprehensive approach to care, as well as consider the impact and influence of a temporary dysphagia versus a more chronic presentation. Finally, the evidence for management of dysphagia in this specialized population is reviewed, highlighting the importance of identifying physiological deficits, feedback, and taking a multidisciplinary approach to care.

${ }^{1}$ Communication Sciences and Disorders, Adelphi University, Garden City, New York; ${ }^{2}$ Speech-Language Pathology, New York Medical College, Valhalla, New York; ${ }^{3}$ Center for Swallowing and Speech-Language Pathology, New York-Presbyterian Brooklyn Methodist Hospital, Brooklyn, New York.

Address for correspondence: Ashwini NamasivayamMacDonald, Ph.D., CCC-SLP, SLP(C), Adelphi University, One South Avenue, HWC 202A, Garden City, NY 11530 (e-mail: ANamaMac@adelphi.edu).
Clinical Decision Making in Dysphagia; Guest Editors, Gary H. McCullough, Ph.D., CCC-SLP and Balaji Rangarathnam, Ph.D., CCC-SLP.

Semin Speech Lang 2019;40:227-242. Copyright (C) 2019 by Thieme Medical Publishers, Inc., 333 Seventh Avenue, New York, NY 10001, USA. Tel: +1(212) 5844662 .

DOI: https://doi.org/10.1055/s-0039-1688837.

ISSN 0734-0478. 
KEYWORDS: deglutition, dysphagia, aging, assessment, treatment

Learning Outcomes: As a result of this activity, the reader will be able to (1) discuss the natural changes of the swallow expected to occur with age; (2) identify feasible screening protocols for use with older adults; (3) explain important considerations when completing clinical bedside evaluations with older adults; and (4) summarize optimal management techniques for use with older adults.

The aging population is increasing at a fast pace around the globe. As of 2012, there was only one country where the proportion of older adults exceeded 30\%: Japan. According to the World Health Organization (WHO), by the middle of this century, many countries will have a similar proportion of older people to that of Japan in 2012. ${ }^{1}$ In the United States, there were approximately 47.8 million people aged 65 and older as of 2015, and this number is expected to grow to 98.2 million by $2060 .^{2}$ This is in line with predictions made by the U.S. Census in 2010. Persons over the age of 65 represent more than $14 \%$ of the total U.S. population. This group has also been growing at a faster rate (15.1\%) than the general U.S. population (9.7\%) since 2000. The impact of this change in demographics has brought with it many challenges for current social, educational, and healthcare systems. Many have paid attention to these changes over the past few decades, but change has been gradual and, some believe, slow.

In 2015, the WHO presented the World Report on Aging and Health, ${ }^{1}$ which outlines a framework focused on looking at aging in terms of functional ability and intrinsic capacity. This is different from our current models which focus on disease-centered care. The WHO report states, "Life expectancy in older age is increasing at a much faster rate in high-income countries than in lower-resource settings, although this varies among specific countries and between males and females" (p. 61). The report also mentions falling frailty rates as a reason for increasing life expectancy. This leads to addressing the question: are added years lived spent in good health or poor health? The answer to this question is quite complex and remains inconclusive. It is unclear if the quality of life in older adults in high-income countries remains good. Countries like the United States have attempted to research this question over the past 30 years; however, the results reported remain inconsistent. Some report falling rates of disability, while others mention an increase in rates of chronic disease and comorbidity. ${ }^{3-6}$ This WHO report on aging and health concludes:

To meet the needs of ageing populations, significant changes are required in the way health systems are structured and health care is delivered. In many places, particularly in low- and middle-income countries, access and affordability are key barriers to care. New services and approaches will need to be developed in these settings.

This suggests that we must reevaluate our speech-language pathology (SLP) practices to meet the needs of older adults. To do this, we must understand the available evidence for optimizing the health of this subset of our population. As such, the primary purpose of this review is to summarize and discuss the current literature regarding best practices for assessing and treating older adults with swallowing impairments. This will be done using a simple guiding framework, while integrating multiple perspectives and strategies to demonstrate the benefits of taking a comprehensive, multidisciplinary approach to care. In doing this, gaps in the literature are also identified to guide future research.

\section{ESTABLISHING A BASELINE FOR SWALLOWING IN OLDER ADULTS}

It is well established that the aging process impacts the structures and function of the 
swallowing tract, which includes the oral, pharyngeal, and esophageal phases of swallow. Throughout this tract, physiological changes may impact bolus flow from the mouth through the esophagus, and ultimately to the gut (stomach). Dysphagia, or swallowing disorder, is very generally defined as an impairment of swallowing safety (i.e., airway invasion) and/ or swallowing efficiency (i.e., residue left behind after the swallow, increased time to swallow, and/or lack of coordination while swallowing). Dysfunction in this tract may increase the risk for aspiration, aspiration pneumonia, malnutrition, and dehydration, to name a few. ${ }^{7-9}$ Among the most common causal factors for dysphagia in older adults are stroke, progressive neurological diseases (e.g., Parkinson's disease, multiple sclerosis), frailty, or dementia. It is known that as we get older, our bodies undergo age-related changes, regardless of the presence of disease or any other underlying medical conditions. In addition to more commonly known changes to eyesight, presbyopia, ${ }^{10}$ and to hearing, presbycusis $^{11}$ and age-related changes to swallowing, or presbyphagia, ${ }^{12}$ are also inevitable. Unlike dysphagia, presbyphagia is generally asymptomatic and is hypothesized to be the result of changes in the anatomy and physiology of the head and neck, muscle loss (sarcopenia), reduced functional reserve, and onset of age-related illness. Very infrequently is presbyphagia mentioned as a causal factor for an acute illness that may result in dysphagia. This may be because someone with presbyphagia remains functional, or asymptomatic, as previously stated, albeit at risk for dysfunction in the presence of weakness or acute illness.

Older adults in general are more vulnerable to disease, and with the increased threat of acute illnesses, medications, and any number of agerelated conditions, they can cross the line from having a healthy older swallow to being a person with oropharyngeal dysphagia. It is critical that clinicians understand that the onset of illness or disease can exacerbate the signs of presbyphagia, increasing the risk of dysphagia. This link between presbyphagia and dysphagia may be realized for some older adults and may never come to fruition for others. As such, until real, functional changes are evident, we must recognize that natural changes are expected and often will still allow for a functional swallow. To compensate for these progressive changes, anecdotal clinical reports suggest that patients adapt their eating habits by taking smaller bites, eating more slowly, and/or avoiding food and liquids that prove to be more taxing to consume. Many of these compensations are not made with the awareness that a swallowing difficulty exists, so by the time an individual seeks the expertise of a swallowing specialist, or SLP, the impairment has reached a level that is no longer manageable and may have other consequences, such as weight loss, respiratory involvement, and/or malnutrition. Another factor to consider, and for which we do not have much supporting evidence or clinical models, is whether the dysfunction in swallow is a temporary one (acute) or a more long-term or progressive one (chronic). For example, when decompensated older adults are evaluated for dysphagia, they may present with dysfunction; yet, once they reach an improved health status, they may return to their baseline swallow function, including their baseline diet. As such, it is important that our clinical assessments are comprehensive, and we adopt a multidisciplinary team approach to care. This means involving other health professionals like dietitians, who can help manage the weight loss and malnutrition, along with geriatricians, pulmonologists, neurologists, and nurses, to name a few.

Specific changes to the anatomy of the swallow for older adults include loss of muscle fibers, more fat in the muscles, and progressive degeneration of the muscles. ${ }^{13}$ More specific to the head and neck musculature, with age we can expect a descending larynx ${ }^{14}$ and increased pharyngeal volume. ${ }^{15}$ The result of these changes in anatomy is changes in swallowing physiology, including increased swallow apnea, ${ }^{16}$ increased oral and pharyngeal pressures exerted during the swallow, ${ }^{17}$ increased incidences of penetration, ${ }^{18,19}$ increased pharyngeal residue, ${ }^{20}$ and delayed initiation in laryngeal and pharyngeal events. ${ }^{21} \mathrm{We}$ also have evidence to suggest that with age we can expect reduced laryngeal excursion, ${ }^{22,23}$ reduced anterior excursion, ${ }^{22,24}$ reduced width of upper esophageal opening, ${ }^{22,25}$ and reduced pharyngeal constriction. ${ }^{26,27}$ A review comparing swallowing timing in healthy young 
and healthy older adults also found that swallow reaction times, pharyngeal delay times, and duration of upper esophageal sphincter opening are longer in healthy older adults. ${ }^{28}$ The review also found that the time from bolus entry into the pharynx to epiglottic deflection tends to be shorter in healthy older adults, but bolus transit parameters and many other swallow timing parameters do not tend to change as a function of age.

\section{CARE SETTINGS: FROM THE COMMUNITY TO LONG-TERM CARE}

A report by the U.S. Department of Health and Human Services found that in 2012, eight million Americans were benefitting from long-term care (LTC), ${ }^{29}$ also known as nursing homes, skilled nursing facilities, dementia care units, or complex continuing care facilities. Given the rapidly increasing global rates of older adults, ${ }^{1}$ one can also expect the number of people residing in LTC facilities to grow. Impaired cognition, which is often the result of dementia in older adults, is a common precipitating factor for transitioning to LTC services. Prior to this transition, older adults often benefit from living at home in the communities they are most familiar with. This results in a significant reduction in mortality rates. ${ }^{30}$ It also allows older adults to maintain informal relationships and interactions, which strengthens ties to their communities and enhances wellbeing, ${ }^{31,32}$ while providing them with feelings of security and familiarity. ${ }^{33}$ Community-dwelling older adults also report fewer swallowing difficulties compared with those residing in LTC. ${ }^{34}$ By contrast, compared with community-dwelling persons, residents in LTC facilities have more functional disabilities complicated by underlying medical illnesses and are at increased risk of acquiring infectious diseases. In 2004, Furman et al reported that pneumonia is the leading cause of morbidity and mortality in this group. ${ }^{35}$ Risk factors included unwitnessed aspiration, sedative medication, and comorbidity. Recognition may be delayed because, in this population, pneumonia often presents without fever, cough, or dyspnea. According to Furman et al, accurate identification of the etiologic agent is "hampe- red because most patients cannot produce a suitable sputum specimen." ${ }^{35}$ It was also reported that pneumonia is associated with significant mortality for up to 2 years. Dementia, a prevalent diagnosis in this setting, is independently related to death rate within the first week after the onset of pneumonia, regardless of treatment for the pneumonia. Documented prevention strategies include vaccination against Streptococcus pneumoniae and influenza on admission to the care facility. ${ }^{35}$ Given this research, it is critical that SLPs and other healthcare professionals work to identify swallowing difficulties as soon as possible to help avoid the negative consequences associated with dysphagia, such as aspiration pneumonia.

\section{SWALLOWING SCREENING}

Fig. 1 displays a simple flow chart outlining the ideal clinical process that patients with risk of dysphagia should move through. This first step in the process is screening patients to help streamline who should receive a clinical swallow evaluation. This is generally a minimally invasive and quick pass/fail procedure. Regardless of living situation, regular swallowing screenings should be performed on all older adults to facilitate the early identification of dysphagia, given older adults' high risk of acquiring aspiration pneumonia. ${ }^{36}$ Research conducted by Hinchey et $\mathrm{al}^{37}$ in the stroke population has demonstrated that the implementation of formal dysphagia screening protocols reduces the risk of pneumonia. The authors also found hospitals with formal screening protocols in place screened all patients, whereas other hospitals screened only patients who they thought to be at increased risk for pneumonia, and this was left up to the discretion of the individual performing the screening. While this study has not been replicated with community-dwelling older adults or residents of LTC, it is likely that the findings extend beyond the initial population tested. Taken together, the findings point to the importance and usefulness of implementing formal screening protocols, which can be executed by trained nursing assistants, nurses, or other healthcare professionals.

Older adults who are living in the community should ideally be screened for dysphagia as 


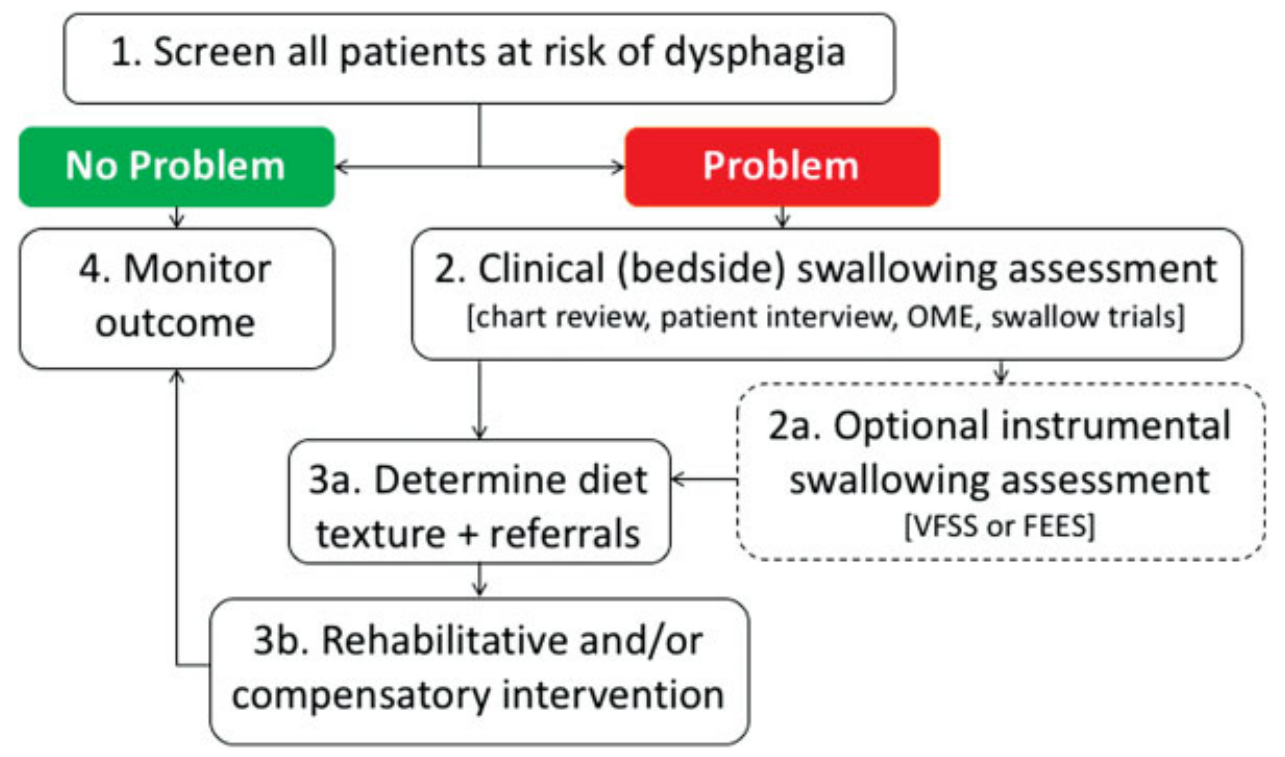

Figure 1 Flowchart depicting ideal patient flow when being screened, assessed, and treated for dysphagia. FEES, fiberoptic endoscopic evaluation of swallowing; OME, oral mechanism exam; VFSS, videofluoroscopic swallow study.

part of their annual physical exam. Currently, there are only three screening protocols validated for use with this population: the Volume Viscosity Swallow Test (V-VST), ${ }^{38}$ the Sydney Swallowing Questionnaire (SSQ), ${ }^{39}$ and the Eating Assessment Tool-10 (EAT-10). ${ }^{40}$ The V-VST involves swallow trials of varying viscosities and volumes, with rules to guide the exact protocol depending on the patient's reaction to each swallow trial. ${ }^{38}$ Although this test is reported to have good sensitivity and specificity for detecting oropharyngeal dysphagia (94 and $88 \%$, respectively $\left.{ }^{41}\right)$, it requires significant training and time to administer; so, it is unlikely to be the first choice for use during regular checkups with a primary care physician. Alternatively, the SSQ involves asking the patient a series of 19 questions, the majority of which are answered using a visual analog scale. ${ }^{39}$ To use the scale, the patient places an "X" on a horizontal line at the point indicating the severity of the dysfunction in question. The sensitivity and specificity of this test have not been documented and the complexity of scoring this test makes it almost as impractical as the V-VST for quick administration and regular use in a medical office setting. The final screening protocol validated for use with community-dwelling older adults and their proxies is the EAT- $10 .^{40}$ This ten-item ques- tionnaire asks patients, or their caregivers, to rate statements detailing their experience with swallowing difficulties on a scale from 0 to 4 , where 0 indicates no problem and 4 indicates a severe problem. The ratings provided for each statement can quickly be added together to produce a final score, where a score greater than 3 indicates that the individual is at high risk of dysphagia. The sensitivity and specificity of this test are also fairly good; 89 and $82 \%$, respectively. ${ }^{40,41}$ This protocol is likely the most clinically feasible for use in a physician's office, as patients or their proxies can fill it out in the waiting room to save time during the visit.

Acknowledging that not all older adults will be seen in a physician's office, the 3-ounce water swallow test has excellent sensitivity (96\%) for identifying patients safe for oral intake within a hospital setting. ${ }^{42}$ It has been validated on patients with a wide variety of medical diagnoses, and can be quickly administered by a nursing assistant or nurse. The patient is provided with a cup filled with 3 ounces of water and is asked to drink all of the water without interruption. A patient is considered to be at high risk for dysphagia if they are unable to drink the entire 3 ounces, if they cough or choke up to 1 minute after completion, and/or present with a wet vocal quality post-swallow. A major benefit of this 
protocol is that it may be easier to administer to patients with mild to moderate cognitive impairments compared with the other screenings previously mentioned. Solely based on ease of administration, the EAT-10 and 3-ounce water swallow test may be the optimal protocols to integrate within a regular health check-up and/ or upon admission to a hospital. The development of clinical protocols incorporating swallowing screenings specifically for use with older adults remains to be seen.

Unfortunately, there also have not been any formal swallowing screening protocols validated for use in an LTC setting. Previous dysphagia research has used a modified version of the Screening Tool for Acute Neurological Dysphagia (also known as the STAND) ${ }^{43}$ to screen residents in LTC for risk of dysphagia. ${ }^{44}$ This modified protocol involves administration of three teaspoons of puree, two dry swallows, and a 3-ounce water swallow test. If any signs or symptoms of aspiration and/or dysphagia (i.e., wet voice, coughing, choking, loss of bolus from mouth, holding bolus in the mouth, delayed/ difficult/painful swallow) were identified at any point during the screening, the protocol was stopped, and the resident was deemed to be at risk of dysphagia. Given that the majority of LTC residents have a dementia diagnosis, ${ }^{45}$ it may also be suitable to use the 3-ounce water swallow test ${ }^{42}$ as a screening protocol in this setting, as it has been validated for use with patients with dementia.

\section{CLINICAL SWALLOW EXAMINATION}

Once those at risk for dysphagia have been identified through failure of the swallow screening, and those with presbyphagia have passed the screening, community-dwelling older adults and LTC residents alike should be referred to a SLP to undergo a swallowing evaluation, which often begins with a clinical swallow evaluation (CSE; Step 2 in Fig. 1). The CSE is sometimes referred to as the bedside examination or the office examination. There are several protocols that can be used to complete a CSE, but the essential components include a chart review, patient/caregiver interview, cranial nerve exam, and swallow trials. ${ }^{46-49}$ Some practitioners also include a language/cognition and speech screening to their CSE protocol. More detailed information on the history and most accepted components can be found in a review article by Riquelme. ${ }^{50} \mathrm{It}$ is important to point out that the reason for a CSE is not just to identify aspiration risk, as it can also provide information on optimal posture, bolus sizes, and other mealtime factors that will ultimately influence swallowing. While SLPs in different facilities and settings use different methods for completing a CSE, we will highlight some of the main considerations when one is assessing older adults.

Background history. To begin, when conducting a chart review for an older adult, the clinician should pay particular attention to any notes on factors known to be independent predictors of aspiration or aspiration pneumonia, as studied by Langmore and colleagues. ${ }^{51}$ The best predictors of aspiration pneumonia for one or more groups of subjects in the study were dependence for feeding, dependence for oral care, decayed teeth, tube feeding, more than one medical diagnosis, taking several medications, and smoking. Langmore et al did a followup study a few years later to consider the predictors of aspiration pneumonia in LTC residents. ${ }^{52}$ The strongest to weakest predictors of aspiration pneumonia were use of suctioning, chronic obstructive pulmonary disease, congestive heart failure, presence of a feeding tube, inability to leave the bed due to illness or weakness, high case mix index (i.e., many resources are allocated to care for/treat the resident), delirium, weight loss, swallowing problems, urinary tract infections (UTIs), modified diet, dependence for eating, dependence in bed (i.e., for moving, turning, etc.), dependence for to move around in general, taking several medications, and increased age. Both of these seminal studies by Langmore et al indicate that dysphagia is an important risk factor for aspiration pneumonia, but generally not enough to cause aspiration pneumonia in the absence of the other risk factors. Considering these factors allows us to have a better understanding of how conservative or liberal we should be in our overall assessment and recommendations. For example, we can be more liberal with patients who only present with dysphagia and none of the other factors shown to be predictive of aspiration 
pneumonia, and more conservative when patients present with several factors in addition to dysphagia, as these patients will have the highest likelihood of developing aspiration pneumonia. If information on these risk factors is limited in the medical chart, they can be probed for during the patient/caregiver interview. It is also relevant to note many physicians may order "aspiration precautions" for patients they consider at risk. Interestingly, in the United States, this order is interpreted differently by different healthcare professionals. There is no national standard for the definition of "aspiration precautions." Healthcare facilities most often employ the term, but do not report on what the definition entails, if they have an agreed-upon definition. This may be part of the reason why we do not have much Level I or Level II evidence for the impact aspiration precautions have on the incidence of aspiration or aspiration pneumonia. ${ }^{53}$

Nutrition. Another factor to consider when completing CSEs with older adults is nutritional status. It is important to note that malnutrition includes those who are dehydrated, and has been negatively associated with physical function and quality of life, and positively associated with risk of institutionalization, hospitalization, and mortality. ${ }^{54}$ Malnutrition reduces one's immune response $^{55}$ and can cause reductions in muscle strength and respiratory function. ${ }^{56}$ It is also associated with fatigue and apathy, which in turn delay recovery. ${ }^{57}$ Reports have indicated that 11 to $37 \%$ of acute care admissions of residents from LTC are avoidable, due to diagnoses such as dehydration and pneumonia. ${ }^{58-60}$ The same two avoidable conditions have been identified as reasons for hospital admission for $13 \%$ of older adults. ${ }^{61}$ Given that many acute care admissions (7\% of LTC residents ${ }^{60}$ and $11 \%$ of older adults ${ }^{61}$ ) are due to dehydration, it is important that SLPs take note of nutritional status. Research has suggested that dysphagia increases the risk of malnutrition for both communitydwelling older adults and those residing in LTC. ${ }^{44,62}$ Given the co-occurrence of, and relationship between, these two conditions, it is critical that both be identified early to avoid hospitalizations and/or increases in hospital stay. If there are no notes in the medical chart on nutritional status, clinicians can probe patients and/or caregivers about recent, unintentional weight loss of $10 \%$ or more of body weight in the previous 6 months. ${ }^{63}$ If unintentional weight loss is occurring, a referral to a dietitian is necessary, as is a thorough CSE to determine if swallowing difficulties are contributing. It is also important to note that recommended daily total fluid intake goals $(3,700 \mathrm{~mL}$ and $2,700 \mathrm{~mL}$ for men and women, respectively) are not being met by the majority of older adults, regardless of place of residence. ${ }^{64,65}$ Inadequate fluid intake may lead to dehydration, and may be the result of dysphagia and/or unpalatable thickened liquids. Dehydration predisposes the person to infections, skin breakdown, hypotension, confusion, and even delirium. All of these may lead to reduced oral intake, which further compromises the person's overall health status. Dehydration may also cause xerostomia (dry mouth), which has been shown to be a contributor toward more rapid colonization of oral bacteria, increasing the risk for an aspiration pneumonia. ${ }^{51}$ Multiple medications may also cause xerostomia and/or a reduction in appetite. ${ }^{51}$ To promote hydration, we should strive to determine the thinnest possible, or least restrictive, liquid consistency that is both safe and efficient. Unfortunately, it is difficult for an SLP to screen for dehydration; so, if any concerns are cited, a referral to a dietitian and/or a discussion with the patient's physician is warranted.

Other related health factors. UTIs have also been associated with dehydration ${ }^{66}$ and are common in LTC settings, accounting for 20 to $30 \%$ of infections as noted by the Division of Healthcare Quality Promotion of the Centers for Disease Control. ${ }^{67}$ Risk factors for developing bacteriuria and UTI include age-related changes to the genitourinary tract, comorbid conditions resulting in neurogenic bladder, and instrumentation required to manage bladder voiding (e.g., catheters). ${ }^{68}$ The need for better understanding and attention to older persons with UTIs by the SLP is often overlooked. The relevance for the SLP stems from overall patient alertness and its potential result in reduced oral (PO) intake. During an active UTI, the patient may need to be fed, which, as previously mentioned, also increases risk for aspirating. ${ }^{51} \mathrm{~A}$ UTI may also contribute to delirium in older adults. ${ }^{69}$ Delirium is often defined as acute, 
transient, usually reversible, fluctuating disturbance in attention, cognition, and consciousness level. $^{70}$ It may last for a few days or a few months. It is known that in older adults with dementia, delirium is less likely to be recognized. Predisposing factors to delirium include cognitive impairment, comorbidity, age, alcohol abuse, sensory impairment, malnutrition, depression, and frailty. ${ }^{71}$ Precipitating factors for delirium include severe illness and infection, restraints, urinary catheter, psychoactive medications, an iatrogenic event, environmental changes, and sleep deprivation. This listing highlights the myriad of risk factors for developing delirium in older adults that may impact oral intake and swallowing status. Additionally, if the patient was already frail, this temporary shift in mental and physical status may well cross the line from presbyphagia to dysphagia and place them at a much greater risk for aspiration, malnutrition, and dehydration.

Interview. When conducting the patient/ caregiver interview to get a thorough case history, it might be helpful to take an ethnographic approach. This approach was first introduced by Westby and originally focused on interviewing parents of children from bilingual homes with possible communication disorders; however, the overall principles apply to the SLP's work and offer ideas on how to present questions in a more open-ended and nonjudgmental manner. ${ }^{72}$ Taking an ethnographic approach includes listening to the behaviors and beliefs that the patient or caregiver reports through a systematic and guided dialogue. Ethnographic interviewing conveys empathy/ acceptance of the world as defined by the informant, allows the clinician to collect necessary information for generating appropriate support and clinical practice, helps equalize the power differential, provides a means for the professional to discover the culture of the family and their strengths and needs, provides a means for focusing on the perspective of the informant, helps reduce potential bias in assessment and intervention, and allows the clinician to collect data in a more ecologically valid framework. ${ }^{72,73}$ In preparation for the ethnographic interview, the clinician has a general set of questions at the outset, but the flow of questioning is molded by the scope and depth of information obtained as the interview unfolds. The clinician must also pay attention to how questions are worded, using open-ended rather than closed-ended questions, using presupposition questions effectively, asking one question at a time, and making use of preliminary statements, while maintaining control of the interview. An example of a question often presented during dysphagia treatment is, "Have you been eating the 4 ounces of puree snacks three times a day that I recommended?" versus a more ethnographically appropriate question: "Tell me what you've been eating by mouth over the last few days." It is anticipated the latter question will provide more relevant information for the clinician working with this older patient.

Another consideration during the patient interview is that patients are not always reliable self-reporters of swallowing impairments. A recent study conducted in LTC demonstrated that residents are largely unable to accurately self-report swallowing difficulties, and also have difficulty accurately reporting incidences of coughing and choking. ${ }^{74}$ More specifically, when asking residents about the presence of swallowing impairments, regardless of level of cognitive function, those who cited difficulties generally were correct, but those who said they had no issues were not always correct in this self-report. These findings suggest that we must not rely on patient reports alone, but must pair these reports with more objective measures of swallowing. We must also educate our patients regarding the signs and symptoms of dysphagia so that they can better recognize a problem when it occurs.

Caregivers. In order for older adults to remain at home for as long as possible and reap the many benefits of doing so, they often require the support of both informal and formal caregivers. Caregivers may assist with basic activities of daily living, such as bathing, meal preparation, and sometimes even feeding. ${ }^{75}$ Some research has suggested that caregivers spend so much time with their care recipients that they can reliably provide information on a care recipient's health status-sometimes more accurately than the care recipients themselves. ${ }^{76}$ Despite the many benefits of older adults living at home, the caregivers often feel burdened by their many responsibilities and the added stress 
of taking care of a loved one. ${ }^{77-80} \mathrm{~A}$ recent systematic review indicated that when older, community-dwelling adults present with swallowing difficulties, caregivers share this burden. ${ }^{81}$ More specifically, spouses of older adults with dysphagia tend to suffer from emotional burden, while adult, child caregivers of aging parents have cited feeling both emotional and physical burden. ${ }^{82,83}$ Clinically, this means that we must learn to identify caregivers who require extra supports and help them care for our patients, so that our patients can reside comfortably at home for as long as possible. In addition to the care recipient presenting with dysphagia, spousal caregivers are at particularly high risk to feel emotionally burdened if the care recipient also has a poor memory and argues with their spouse, and if the caregiver is female, has attained a relatively high level of education, has their own health issues, and needs to help the care recipient with personal care. $^{82}$ Similarly, child caregivers of aging parents with swallowing difficulties are most likely to feel emotionally burdened if the care recipient has osteoporosis, a history of falls and/ or argues with their child, and if the caregiver has achieved a relatively high level of education, helps the care recipient for several hours per day, and has disagreements with other family members in regard to their parent's care. ${ }^{83}$ These child caregivers are also likely to suffer from physical burden when their parent has dysphagia, has had a heart attack and/or has been diagnosed with heart disease, and if the caregiver is a female with health issues, helps their parent with personal care, and disagrees with other family members in regard to their parent's care. ${ }^{83}$ Once informal caregivers who are at high risk of feeling burdened are identified, we can provide basic education on the management of swallowing difficulties. This is central to relieving some burden, as few caregivers feel like they receive adequate support and education on how to best manage their care recipient's dysphagia. ${ }^{84,85}$ This may be due to the fact that as healthcare professionals we assume that spouses and children are more than capable of carrying out their caregiving duties ${ }^{86}$ and do not ask about what additional information or resources they may require. Anecdotal clinical reports also suggest that we do not check-in with caregivers as we might with our patients, and research has suggested that the information we provide to caregivers is highly medicalized and generic. ${ }^{87}$ Therefore, to allow our patients to live comfortably at home for as long as possible, we need to support their caregivers-emotionally and physically-as best as we can, including exploring concerns regarding the prognosis of the progressing, or temporary, dysphagia, and plans for maintaining good nutritional status. This may also mean we need to involve other members of the care team in educating and providing caregivers with appropriate resources when necessary.

\section{INSTRUMENTAL EXAMINATIONS}

After the CSE has been completed and dysphagia has been identified, the SLP needs to decide if an instrumental examination is warranted (Step 2a in Fig. 1). It is relevant to mention that the referring clinician should understand the purpose of the instrumental examination, and when to best refer the patient for one. A videofluoroscopic swallow study (VFSS) or a fiberoptic endoscopic evaluation of swallowing (FEES) exam might be performed when the results of the CSE are inconclusive or incomplete in terms of location and reason for dysphagia, and/or to identify any physiological impairments to form a comprehensive treatment plan. These instrumental examinations, however, capture a short period of time in comparison to the entire mealtime experience, and thus should be included as a portion of the decision-making process for the clinician. Like the CSE, there are several protocols that can be used to conduct these instrumental assessments. Ultimately, stimuli administered need to be chosen carefully to demonstrate the patient's abilities and impairments, as well as stress the swallowing system to help determine the patient's limits. When conducting these assessments with older adults, it is important to bear in mind the swallowing physiology and signs of dysphagia considered to be within the range of normal for older adults. For example, Penetration-Aspiration Scale (PAS) scores of 2, indicating airway invasion above the level of the vocal folds 
with subsequent successful ejection of the bolus, with thin liquids are not uncommon for adults over the age of 63 years. ${ }^{12}$ PAS scores of 3 , indicating airway invasion with material remaining above the level of the vocal folds, are also said to occur in $16.5 \%$ of healthy older adults' swallows. ${ }^{12}$ Daggett et $\mathrm{al}^{18}$ had similar findings: the frequency of penetration for thin liquid and semisolid boluses was $17.4 \%$ in healthy adults over the age of 50 years, compared with $6.8 \%$ in younger adults. We also know that significantly more penetration will occur with larger liquid boluses and is less common for solid boluses and boluses more viscous than water. ${ }^{18}$ Penetration is more frequent of the first trial of a given consistency during videofluoroscopy. ${ }^{12}$ These are all characteristics of presbyphagia, rather than dysphagia. Consequently, we should be weary of making diet changes based on findings of penetration alone or on one trial only. Other research has suggested that piecemeal deglutition, premature loss of liquid, and oral and pharyngeal residue are significantly more common in older adults compared with their younger counterparts. ${ }^{88}$ Moreover, the oral preparatory phase of swallowing, ${ }^{89}$ oral transit time, swallow reaction time, and pharyngeal transit time have also been found to be significantly longer in older adults. ${ }^{88}$ It is important to note that there is no convincing evidence to suggest that these age-related changes in swallow function are due to reduced functional reserve. However, it is likely that the increased frequency of airway invasion that occurs in healthy adults is a consequence of presbyphagia-namely, longer swallow reaction times ${ }^{28}$ and a more inferior larynx. ${ }^{14}$

In addition to these characteristic changes associated with presbyphagia, SLPs must also consider the physiological factors that have been identified as related to aspiration risk. A systematic review by Steele and Cichero ${ }^{90}$ identified several measures that are reported to demonstrate an association with increased risk of airway invasion. For instance, reduced maximum isometric pressures, reduced swallowing tongue pressures, and reduced tongue driving force were identified as factors associated with aspiration. Reduced anterior hyoid movement (as measured in normalized units) and longer bolus dwell time in the pharynx while the laryngeal vestibule was open were also associated with greater risk of airway invasion, whereas duration of laryngeal vestibule closure was not identified as being able to differentiate between aspirators and nonaspirators. Lastly, a respiratory rate of less than 25 breaths/minute, a low baseline oxygen saturation level $\left(<94 \% \mathrm{SpO}_{2}\right)$, and a respiratory pattern that differed from the typical exhale-swallow-exhale pattern were all associated with increased risk of aspiration. Therefore, if aspiration is observed in the presence of one or more of these factors, one might better understand the underlying mechanism for the swallowing impairment and select an intervention that addresses the factor(s) at play.

\section{DYSPHAGIA MANAGEMENT}

Once the diagnostic workup has been completed, it is time for the clinician to determine a diet texture that maximizes both swallow safety and efficiency, decide on any treatment approaches to be employed, as well as make any necessary referrals to other healthcare professionals (Step $3 \mathrm{a}$ in Fig. 1). Appropriate rehabilitative and/or compensatory techniques (Step 3b of Fig. 1) need to be chosen based on the previously identified impairments, the patient's medical history, including his/her functional abilities, and the setting in which the patient is being seen. Dysphagia management in the acute hospital setting primarily focuses on compensatory techniques and regular monitoring for change in status. These compensatory techniques often include diet texture modifications, ${ }^{91}$ changes in posture, changes in bolus size, and clearing swallows. In other settings, rehabilitation of the swallow is more common. This allows for the impaired physiology to be targeted within treatment sessions in hopes of a long-term change in swallowing ability. It is important to keep in mind that more frequent therapy sessions will result in better chances of regaining functional swallowing abilities, as well as fewer dysphagia-related medical complications (primarily aspiration pneumonia), less mortality, and reduced need for LTC. ${ }^{92}$ While many swallowing exercises have been trialed on healthy older adults or in specific populations (e.g., stroke, Parkinson's disease, and head and neck cancer), fewer have been identified for use with the general community- 
dwelling older adult population and those residing in LTC. One such exercise program that has been trialed with older adults is tongue strengthening, an 8-week program has been shown to increase both isometric and swallowing pressures. ${ }^{93}$ Tongue strength training has also been piloted in LTC; the researchers found that residents were able to participate and show improvements in tongue strength, ${ }^{94}$ which may improve post-swallow vallecular residue with thin liquids. ${ }^{95}$ Integrating effortful swallows into rehabilitation protocols with older adults has also been proven to be feasible and effective. Research has demonstrated that this type of swallow serves to increase maximum anterior hyoid excursion, laryngeal vestibule closure, and duration of upper esophageal sphincter opening. ${ }^{96}$ There was also a trend of reduced oral residue when the effortful swallow was implemented. Expiratory muscle strength training is another method of rehabilitation that appears promising when used with older adults. A 4week program significantly increased maximum expiratory pressure, reflecting an increased expiratory force generating capacity comparable with findings in healthy young adults. ${ }^{97}$ Most recently, the head lift exercise (commonly referred to as the Shaker exercise) and the recline exercise were found to be equally effective in improving superior and anterior hyoid excursion in older adults, though the recline exercise required significantly less effort. ${ }^{98}$ Despite a lack of strong evidence for the use of many other swallowing exercises and techniques with older adults, such as neuromuscular electrical stimulation, ${ }^{99}$ SLPs should consider that there are rehabilitation strategies other than the ones previously mentioned that may be effective in changing swallow physiology when used with older adults, such as the Mendelsohn maneuver, ${ }^{100}$ supraglottic swallow, ${ }^{101}$ and the Masako (also known as the tongue hold swallow). ${ }^{102}$ Ultimately, in addition to the available evidence, one should consider the patient's physiological impairment and, ideally, test the efficacy of a specific exercise via an instrumental exam.

An additional consideration when developing a treatment protocol for a patient is the use of biofeedback. While much of the research using biofeedback mechanisms has been conducted with the stroke population, there is fairly strong evidence to suggest that biofeedback can be used as an effective adjunct to swallow therapy with patients who present with pharyngeal dysphagia. ${ }^{103-106}$ Exercise science literature suggests that biofeedback is most useful when patients are learning new and/or unfamiliar tasks. ${ }^{107}$ The most commonly used method of feedback discussed in the literature is surface electromyography (sEMG), which is a noninvasive and relatively inexpensive technique that allows both the clinician and patient to monitor muscle activity during task-specific exercises. For patients who have intact cognition, this allows for the provision of immediate auditory and/or visual feedback on the accuracy, degree of muscle contraction, and timing of the swallowing exercise, ${ }^{108}$ which in turn can improve functional swallowing ability. ${ }^{97}$ However, regardless of the use of equipment-based feedback, like sEMG, it is important to recognize that clinician belief and enthusiasm in a treatment will impact patient buy-in and may positively affect treatment outcomes. ${ }^{109}$

Interestingly, given what is known about presbyphagia, there has been very little research conducted to elucidate the role of preventative/ prophylactic exercises in this population. One recent study by Balou and colleagues ${ }^{110}$ found significant improvements in swallowing physiology after an 8-week comprehensive swallowing treatment protocol in a small group of healthy adults who had at least one swallow with a safety impairment (PAS score $>2$ ) or at least one swallow with an efficiency impairment (Modified Barium Swallow Impairment Profile ${ }^{111}$ Component 16 Pharyngeal Residue score $>1$ ). The description of the participants suggests that they presented with presbyphagia rather than dysphagia, indicating that perhaps the condition can be reversed. This is in line with the theory that presbyphagia and dysphagia lie on a continuum, and we slowly move through this continuum as we age. ${ }^{20}$ Another study by Robbins and colleagues found that tongue pressure training in healthy older adults resulted in improvements in tongue strength and peak swallow pressures. ${ }^{93}$ However, as alluded to previously, there is no strong evidence that improvements in tongue strength result in positive changes to swallowing function. As such, additional research is needed to confirm if we can indeed decrease the risk of 
dysphagia by reducing the consequences of presbyphagia.

Regardless of the subset of older adults being treated, when developing an individualized dysphagia management plan for older patients, many of the factors discussed in the swallowing assessment sections of this article should also be integrated into treatment. This includes understanding the patient's perception of the problem, providing caregiver education to reduce their feelings of burden, involving a dietitian if adequate nutrition is a concern, taking into consideration possible delirium, and, of course, being culturally sensitive. This approach also fits into the WHO International Classification of Functioning, Disability and Health framework ${ }^{112}$ that addresses functioning and disability related to a health condition within the context of the individual's activities and participation in everyday life. Like the framework suggests, our goal is to optimize each patient's potential to participate in meaningful activities-like eating and drinkingwhile maximizing the outcomes that lead to functional improvements that are important to the patient. While integrating these many factors into a dysphagia management plan may seem daunting, leaning on the multidisciplinary team is crucial to providing optimal care without overwhelming one single clinician.

\section{CONCLUSION}

Given the growing aging population, it is very likely that the SLP working with adults will encounter an increased caseload of persons over the age of 65 years. Providing patient-centered dysphagia assessment, treatment, and management services to this population requires a different clinical skill-set-one that includes an integration of the latest findings in swallow pathophysiology into clinical practice, along with a thorough understanding of the multifaceted social, economic, and cultural factors that influence the person's overall health status. It should be expected that the SLP working with the growing aging population remains current with the latest findings in expected and unexpected changes in swallow physiology. Designing and integrating clinical protocols that differ for the aging population is the only way to move toward ensuring the delivery of services that are age appropriate, least-restrictive, and may serve to improve quality of life while helping contain healthcare costs. Much more research and multidisciplinary collaborations are needed to further understand the complexities and challenges of caring for the older adult, including the many potential comorbidities impacting care. The SLP should never underestimate the role we play in influencing quality of life and healthcare economics when providing good clinical care. This is essential as we strive to achieve clinically and culturally relevant outcomes in dysphagia care for older adults around the globe.

\section{CONFLICTS OF INTEREST}

The authors have no conflicts of interest to disclose.

\section{REFERENCES}

1. World Health Organization. World Report on Ageing and Health. World Health Organization; 2015

2. United States Census Bureau. Older Americans Month: May 2017. Available at: https://www. census.gov/content/dam/Census/newsroom/ facts-for-features/2017/cb17-ff08.pdf. Accessed January 14, 2019

3. Alecxih L, Shen S, Chan I, Taylor D, Drabek J. Individuals Living in the Community with Chronic Conditions and Functional Limitations: $\mathrm{A}$ Closer Look. U.S. Department of Health and Human Services; 2010. Available at: http://aspe. hhs.gov/daltcp/reports/2010/closerlook.pdf. Accessed April 24, 2019

4. Balarajan Y, Selvaraj S, Subramanian SV. Health care and equity in India. Lancet 2011;377 (9764):505-515

5. Fitzpatrick AL, Powe NR, Cooper LS, Ives DG, Robbins JA. Barriers to health care access among the elderly and who perceives them. Am J Public Health 2004;94(10):1788-1794

6. Terraneo M. Inequities in health care utilization by people aged $50+$ : evidence from 12 European countries. Soc Sci Med 2015;126:154-163

7. Hudson HM, Daubert CR, Mills RH. The interdependency of protein-energy malnutrition, aging, and dysphagia. Dysphagia 2000;15(01): 31-38

8. Mendez L, Friedman LS, Castell DO. Swallowing disorders in the elderly. Clin Geriatr Med 1991;7(02):215-230 
9. Mion LC, McDowell JA, Heaney LK. Nutritional assessment of the elderly in the ambulatory care setting. Nurse Pract Forum 1994;5(01):46-51

10. Glasser A, Campbell MC. Presbyopia and the optical changes in the human crystalline lens with age. Vision Res 1998;38(02):209-229

11. Gates GA, Mills JH. Presbycusis. Lancet 2005; 366(9491):1111-1120

12. Robbins J, Hamilton JW, Lof GL, Kempster GB. Oropharyngeal swallowing in normal adults of different ages. Gastroenterology 1992;103(03):823-829

13. Evans WJ, Lexell J. Human aging, muscle mass, and fiber type composition. J Gerontol A Biol Sci Med Sci 1995;50(Special Issue):11-16

14. Angerstein W. [Vocal changes and laryngeal modifications in the elderly (presbyphonia and presbylarynx)]. Laryngorhinootologie 2018;97 (11):772-776

15. Molfenter SM, Amin MR, Branski RC, et al. Age-related changes in pharyngeal lumen size: a retrospective MRI analysis. Dysphagia 2015;30 (03):321-327

16. Leslie P, Drinnan MJ, Ford GA, Wilson JA. Swallow respiratory patterns and aging: presbyphagia or dysphagia? J Gerontol A Biol Sci Med Sci 2005;60(03):391-395

17. Shaker R, Lang IM. Effect of aging on the deglutitive oral, pharyngeal, and esophageal motor function. Dysphagia 1994;9(04):221-228

18. Daggett A, Logemann J, Rademaker A, Pauloski B. Laryngeal penetration during deglutition in normal subjects of various ages. Dysphagia 2006; 21(04):270-274

19. Butler SG, Stuart A, Markley L, Rees C. Penetration and aspiration in healthy older adults as assessed during endoscopic evaluation of swallowing. Ann Otol Rhinol Laryngol 2009;118(03):190-198

20. de Lima Alvarenga EH, Dall'Oglio GP, Murano $\mathrm{EZ}$, Abrahão M. Continuum theory: presbyphagia to dysphagia? Functional assessment of swallowing in the elderly. Eur Arch Otorhinolaryngol 2018;275(02):443-449

21. Logemann JA, Kahrilas PJ, Kobara M, Vakil NB. The benefit of head rotation on pharyngoesophageal dysphagia. Arch Phys Med Rehabil 1989;70 (10):767-771

22. Logemann JA, Pauloski BR, Rademaker AW, Colangelo LA, Kahrilas PJ, Smith CH. Temporal and biomechanical characteristics of oropharyngeal swallow in younger and older men. J Speech Lang Hear Res 2000;43(05):1264-1274

23. Logemann JA, Pauloski BR, Rademaker AW, Kahrilas $\mathrm{PJ}$. Oropharyngeal swallow in younger and older women: videofluoroscopic analysis. J Speech Lang Hear Res 2002;45(03):434-445

24. Kim Y, McCullough GH. Maximum hyoid displacement in normal swallowing. Dysphagia 2008; 23(03):274-279
25. Kern M, Bardan E, Arndorfer R, Hofmann C, Ren J, Shaker R. Comparison of upper esophageal sphincter opening in healthy asymptomatic young and elderly volunteers. Ann Otol Rhinol Laryngol 1999;108(10):982-989

26. Leonard R, Kendall KA, McKenzie S. Structural displacements affecting pharyngeal constriction in nondysphagic elderly and nonelderly adults. Dysphagia 2004;19(02):133-141

27. Kendall KA, Leonard RJ. Pharyngeal constriction in elderly dysphagic patients compared with young and elderly nondysphagic controls. Dysphagia 2001;16(04):272-278

28. Namasivayam-MacDonald AM, Barbon CEA, Steele CM. A review of swallow timing in the elderly. Physiol Behav 2018;184:12-26

29. Harris-Kojetin L, Sengupta M, Park-Lee E, Valverde R. Long-term care services in the United States: 2013 overview. Vital Health Stat 3 2013; (37):1-107

30. Elkan R, Kendrick D, Dewey M, et al. Effectiveness of home based support for older people: systematic review and meta-analysis. BMJ 2001; 323(7315):719-725

31. Gardner PJ. Natural neighborhood networks important social networks in the lives of older adults aging in place. J Aging Stud 2011;25(03):263-271

32. Sabia JJ. There's no place like home: a hazard model analysis of aging in place among older homeowners in the PSID. Res Aging 2008;30(01):3-35

33. Wiles JL, Leibing A, Guberman N, Reeve J, Allen $\mathrm{RE}$. The meaning of "aging in place" to older people. Gerontologist 2012;52(03):357-366

34. Madhavan A, LaGorio LA, Crary MA, Dahl WJ, Carnaby GD. Prevalence of and risk factors for dysphagia in the community dwelling elderly: a systematic review. J Nutr Health Aging 2016;20 (08):806-815

35. Furman CD, Rayner AV, Tobin EP. Pneumonia in older residents of long-term care facilities. Am Fam Physician 2004;70(08):1495-1500

36. Marik PE, Kaplan D. Aspiration pneumonia and dysphagia in the elderly. Chest 2003;124(01): 328-336

37. Hinchey JA, Shephard T, Furie K, Smith D, Wang D, Tonn S; Stroke Practice Improvement Network Investigators. Formal dysphagia screening protocols prevent pneumonia. Stroke 2005; 36(09):1972-1976

38. Rofes L, Arreola V, Clavé P. The volume-viscosity swallow test for clinical screening of dysphagia and aspiration. In: Cichero J, Clavé P, eds. Stepping Stones to Living Well with Dysphagia. Vol 72. Karger Publishers; 2012:33-42

39. Wallace KL, Middleton S, Cook IJ. Development and validation of a self-report symptom inventory to assess the severity of oral-pharyngeal dysphagia. Gastroenterology 2000;118(04):678-687 
40. Belafsky PC, Mouadeb DA, Rees CJ, et al. Validity and reliability of the Eating Assessment Tool (EAT-10). Ann Otol Rhinol Laryngol 2008;117 (12):919-924

41. Rofes L, Arreola V, Mukherjee R, Clavé P. Sensitivity and specificity of the Eating Assessment Tool and the Volume-Viscosity Swallow Test for clinical evaluation of oropharyngeal dysphagia. Neurogastroenterol Motil 2014;26(09):1256-1265

42. Suiter DM, Leder SB. Clinical utility of the 3ounce water swallow test. Dysphagia 2008;23(03): 244-250

43. Shephard T. Dysphagia update: evidence, tools, and practice. Presented at the International Stroke Conference; 2007; San Francisco, CA

44. Namasivayam-MacDonald AM, Morrison JM, Steele CM, Keller H. How swallow pressures and dysphagia affect malnutrition and mealtime outcomes in long-term care. Dysphagia 2017;32 (06):785-796

45. Keller HH, Carrier N, Slaughter SE, et al. Prevalence and determinants of poor food intake of residents living in long-term care. J Am Med Dir Assoc 2017;18(11):941-947

46. Martino R, Pron G, Diamant N. Screening for oropharyngeal dysphagia in stroke: insufficient evidence for guidelines. Dysphagia 2000;15(01):19-30

47. McCullough GH, Wertz RT, Rosenbek JC. Sensitivity and specificity of clinical/bedside examination signs for detecting aspiration in adults subsequent to stroke. J Commun Disord 2001;34 (1-2):55-72

48. McCullough GH, Wertz RT, Rosenbek JC, Dinneen C. Clinicians' preferences and practices in conducting clinical/bedside and videofluoroscopic swallowing examinations in an adult, neurogenic population. Am J Speech Lang Pathol 1999; 8(02):149-163

49. Carnaby G. Food for thought: importance of a clinical exam/cranial nerve assessment. Perspect Swallowing Swallowing Disord Dysphagia 2012; 21(04):143-149

50. Riquelme LF. Clinical swallow examination (CSE): Can we talk? Perspect Swallowing Swallowing Disord Dysphagia 2015;24(01):34-39

51. Langmore SE, Terpenning MS, Schork A, et al. Predictors of aspiration pneumonia: how important is dysphagia? Dysphagia 1998;13(02):69-81

52. Langmore SE, Skarupski KA, Park PS, Fries BE. Predictors of aspiration pneumonia in nursing home residents. Dysphagia 2002;17(04):298-307

53. Riquelme LF, Soyfer A, Benjamin R. Presbyphagia: what we need to know to provide best practice. EHearsay J Ohio Speech-Lang-Hear Assoc 2016; 6(01):23-29

54. Marshall S, Bauer J, Isenring E. The consequences of malnutrition following discharge from rehabilitation to the community: a systematic review of current evidence in older adults. J Hum Nutr Diet 2014;27(02):133-141

55. Scrimshaw NS, SanGiovanni JP. Synergism of nutrition, infection, and immunity: an overview. Am J Clin Nutr 1997;66(02):464S-477S

56. Allison SP. Malnutrition, disease, and outcome. Nutrition 2000;16(7-8):590-593

57. Kubrak C, Jensen L. Malnutrition in acute care patients: a narrative review. Int J Nurs Stud 2007; 44(06):1036-1054

58. Carter MW. Factors associated with ambulatory care-sensitive hospitalizations among nursing home residents. J Aging Health 2003;15(02):295-331

59. Intrator O, Zinn J, Mor V. Nursing home characteristics and potentially preventable hospitalizations of long-stay residents. J Am Geriatr Soc 2004;52(10):1730-1736

60. Walker JD, Teare GF, Hogan DB, Lewis S, Maxwell CJ. Identifying potentially avoidable hospital admissions from Canadian long-term care facilities. Med Care 2009;47(02):250-254

61. Nyweide DJ, Anthony DL, Bynum JP, et al. Continuity of care and the risk of preventable hospitalization in older adults. JAMA Intern Med 2013;173(20):1879-1885

62. Takeuchi K, Aida J, Ito K, Furuta M, Yamashita Y, Osaka K. Nutritional status and dysphagia risk among community-dwelling frail older adults. J Nutr Health Aging 2014;18(04):352-357

63. McWhirter JP, Pennington CR. Incidence and recognition of malnutrition in hospital. BMJ 1994;308(6934):945-948

64. Namasivayam-MacDonald AM, Slaughter SE, Morrison J, et al. Inadequate fluid intake in long term care residents: prevalence and determinants. Geriatr Nurs 2018;39(03):330-335

65. Zizza CA, Ellison KJ, Wernette CM. Total water intakes of community-living middle-old and oldest-old adults. J Gerontol A Biol Sci Med Sci 2009;64(04):481-486

66. Pals JK, Weinberg AD, Beal LF, Levesque PG, Cunningham TJ, Minaker KL. Clinical triggers for detection of fever and dehydration. Implications for long-term care nursing. J Gerontol Nurs 1995;21(04):13-19

67. Centers for Disease Control and Prevention. Urinary Tract Infection (UTI) Event for LongTerm Care Facilities. 2015

68. Ribera MC, Pascual R, Orozco D, Pérez Barba C, Pedrera V, Gil V. Incidence and risk factors associated with urinary tract infection in diabetic patients with and without asymptomatic bacteriuria. Eur J Clin Microbiol Infect Dis 2006;25 (06):389-393

69. Eriksson I, Gustafson Y, Fagerström L, Olofsson B. Urinary tract infection in very old women is associated with delirium. Int Psychogeriatr 2011; 23(03):496-502 
70. Beers MH, Fletcher AJ, Jones TV, Porter R, Berkwitz M, Kaplan JL. The Merck Manual of Medical Information. New York, NY: Pocket Books; 2003

71. Ahmed S, Leurent B, Sampson EL. Risk factors for incident delirium among older people in acute hospital medical units: a systematic review and meta-analysis. Age Ageing 2014;43(03):326-333

72. Westby CE. Ethnographic interviewing: asking the right questions to the right people in the right ways. J Childhool Commun Disord 1990;13(01): 101-111

73. Westby C, Burda A, Mehta Z. Asking the right questions in the right ways: strategies for ethnographic interviewing. ASHA Lead 2003;8(08):4-17

74. Namasivayam-MacDonald AM, Steele CM, Keller HH. Swallow function and perception of dysphagia in residents of long-term care. Am J Speech Lang Pathol, In press

75. Tennstedt SL, McKinlay JB, Sullivan LM. Informal care for frail elders: the role of secondary caregivers. Gerontologist 1989;29(05):677-683

76. McPherson CJ, Addington-Hall JM. Judging the quality of care at the end of life: can proxies provide reliable information? Soc Sci Med 2003; 56(01):95-109

77. Diehl-Schmid J, Schmidt E-M, Nunnemann S, et al. Caregiver burden and needs in frontotemporal dementia. J Geriatr Psychiatry Neurol 2013; 26(04):221-229

78. Gallicchio L, Siddiqi N, Langenberg P, Baumgarten M. Gender differences in burden and depression among informal caregivers of demented elders in the community. Int J Geriatr Psychiatry 2002;17(02):154-163

79. Huang SS, Lee MC, Liao YC, Wang WF, Lai TJ. Caregiver burden associated with behavioral and psychological symptoms of dementia (BPSD) in Taiwanese elderly. Arch Gerontol Geriatr 2012; 55(01):55-59

80. Juntunen K, Salminen A-L, Törmäkangas $T$, Tillman P, Leinonen K, Nikander R. Perceived burden among spouse, adult child, and parent caregivers. J Adv Nurs 2018;74(10):2340-2350

81. Namasivayam-MacDonald AM, Shune SE. The burden of dysphagia on family caregivers of the elderly: a systematic review. Geriatrics 2018;3(02): 30

82. Shune SE, Namasivayam-MacDonald AM. Swallowing impairments increase emotional burden in spousal caregivers of older adults. J Appl Gerontol 2019. Doi: 10.1177/0733464818821787. [Epub ahead of print]

83. Shune SE, Namasivayam-MacDonald AM. Dysphagia as an independent risk factor among caregivers of aging parents. Presented at the 27th Annual Dysphagia Research Society Meeting, San Diego, CA; 2019
84. Mayre-Chilton KM, Talwar BP, Goff LM. Different experiences and perspectives between head and neck cancer patients and their care-givers on their daily impact of a gastrostomy tube. J Hum Nutr Diet 2011;24(05):449-459

85. Penner JL, McClement S, Lobchuk M, Daeninck P. Family members' experiences caring for patients with advanced head and neck cancer receiving tube feeding: a descriptive phenomenological study. J Pain Symptom Manage 2012;44(04):563-571

86. Glajchen M. The emerging role and needs of family caregivers in cancer care. J Support Oncol 2004;2(02):145-155

87. Nund RL, Ward EC, Scarinci NA, Cartmill B, Kuipers P, Porceddu SV. Carers' experiences of dysphagia in people treated for head and neck cancer: a qualitative study. Dysphagia 2014;29 (04):450-458

88. Yoshikawa M, Yoshida M, Nagasaki T, et al. Aspects of swallowing in healthy dentate elderly persons older than 80 years. J Gerontol A Biol Sci Med Sci 2005;60(04):506-509

89. Sonies BC, Parent LJ, Morrish K, Baum BJ. Durational aspects of the oral-pharyngeal phase of swallow in normal adults. Dysphagia 1988;3 (01):1-10

90. Steele CM, Cichero JA. Physiological factors related to aspiration risk: a systematic review. Dysphagia 2014;29(03):295-304

91. Cichero JA, Steele C, Duivestein J, et al. The need for international terminology and definitions for texture-modified foods and thickened liquids used in dysphagia management: foundations of a global initiative. Curr Phys Med Rehabil Rep 2013; 1:280-291

92. Carnaby G, Hankey GJ, Pizzi J. Behavioural intervention for dysphagia in acute stroke: a randomised controlled trial. Lancet Neurol 2006;5 (01):31-37

93. Robbins J, Gangnon RE, Theis SM, Kays SA, Hewitt AL, Hind JA. The effects of lingual exercise on swallowing in older adults. J Am Geriatr Soc 2005;53(09):1483-1489

94. Namasivayam-MacDonald AM, Burnett L, Nagy A, Waito AA, Steele CM. Effects of tongue strength training on mealtime function in longterm care. Am J Speech Lang Pathol 2017;26(04): 1213-1224

95. Steele CM, Bayley MT, Peladeau-Pigeon M, et al. A randomized trial comparing two tongue-pressure resistance training protocols for post-stroke dysphagia. Dysphagia 2016;31(03): 452-461

96. Hind JA, Nicosia MA, Roecker EB, Carnes ML, Robbins J. Comparison of effortful and noneffortful swallows in healthy middle-aged and older adults. Arch Phys Med Rehabil 2001;82(12): 1661-1665 
97. Kim J, Davenport P, Sapienza C. Effect of expiratory muscle strength training on elderly cough function. Arch Gerontol Geriatr 2009;48(03):361-366

98. Fujiki RB, Oliver AJ, Malandraki JB, Wetzel D, Craig BA, Malandraki GA. The recline and head lift exercises: a randomized clinical trial comparing biomechanical swallowing outcomes and perceived effort in healthy older adults. J Speech Lang Hear Res 2019;62(03):631-643

99. Clark H, Lazarus C, Arvedson J, Schooling T, Frymark T. Evidence-based systematic review: effects of neuromuscular electrical stimulation on swallowing and neural activation. Am J Speech Lang Pathol 2009;18(04):361-375

100. Logemann JA, Kahrilas PJ. Relearning to swallow after stroke-application of maneuvers and indirect biofeedback: a case study. Neurology 1990;40(07): 1136-1138

101. Logemann JA, Rademaker AW, Pauloski BR, Kahrilas PJ. Effects of postural change on aspiration in head and neck surgical patients. Otolaryngol Head Neck Surg 1994;110(02):222-227

102. Masako F, Logemann JA. Effect of a tongueholding maneuver on posterior pharyngeal wall movement during deglutition. Am J Speech Lang Pathol 1996;5(01):23-30

103. Crary Michael A, Groher Michael E. Basic concepts of surface electromyographic biofeedback in the treatment of dysphagia: a tutorial. Am J Speech Lang Pathol 2000;9(02):116-125

104. Athukorala RP, Jones RD, Sella O, Huckabee ML. Skill training for swallowing rehabilitation in patients with Parkinson's disease. Arch Phys Med Rehabil 2014;95(07):1374-1382

105. Crary MA. A direct intervention program for chronic neurogenic dysphagia secondary to brainstem stroke. Dysphagia 1995;10(01):6-18

106. Huckabee ML, Cannito MP. Outcomes of swallowing rehabilitation in chronic brainstem dysphagia: a retrospective evaluation. Dysphagia 1999; 14(02):93-109

107. Mulder T, Hulstyn W. Sensory feedback therapy and theoretical knowledge of motor control and learning. Am J Phys Med 1984;63(05):226-244

108. Huckabee M-L, Macrae P. Rethinking rehab: skill-based training for swallowing impairment. Perspect Swallowing Swallowing Disord Dysphagia 2014;23(01):46-53

109. Skrabanek P, McCormick J. Follies \& Fallacies in Medicine. Eastbourne, East Sussex: Prometheus Books 1990

110. Balou M, Herzberg EG, Kamelhar D, Molfenter SM. An intensive swallowing exercise protocol for improving swallowing physiology in older adults with radiographically confirmed dysphagia. Clin Interv Aging 2019;14:283-288

111. Martin-Harris B, Logemann JA, McMahon S, Schleicher M, Sandidge J. Clinical utility of the modified barium swallow. Dysphagia 2000;15(03): 136-141

112. Bruyère SM, Van Looy SA, Peterson DB. The international classification of functioning, disability and health: contemporary literature overview. Rehabil Psychol 2005;50(02):113 